\title{
Rheo-Optical Studies of High Polymers. XXI. The Deformation Process and Crystal Transformation in Polybutene-1*
}

\author{
Tadahiro AsAdA, Juichi SASADA and Shigeharu ONOGI** \\ Department of Polymer Chemistry, Kyoto University, \\ Kyoto, Japan. \\ (Received September 7, 1971)
}

\begin{abstract}
The crystal transformation from modification 2 to modification 1 in unstrained polybutene- 1 films was observed by the infrared absorption and refractive index methods. The crystal transformation and orientation during stress-strain measurements were also observed by means of the infrared dichroism method developed by us. The crystal transformation in a fresh sample begins at about 30 min after the sample preparation, and the degree of transformation increases with increasing time until it reaches about 0.9 at $50 \mathrm{hr}$ and 1.0 about 3 weeks after the sample preparation. The rate of crystal transformation could be expressed by an equation of the same form as that for the one-dimensional crystallization rate.

Stress-strain curves, degree of crystal transformation vs. strain curves as well as infrared dichroic ratio vs. strain curves for film specimens having different initial degrees of crystal transformation differ remarkably. On the basis of these experimental results, the deformation and crystal transformation processes during stree-strain measurements under constant rate of elongation are discussed.

KEY WORDS Crystal Transformation / Orientation / Infrared Dichroism / Deformation Mechanism / Rheo-Optical Properties / Polybutene-1 /
\end{abstract}

It is well-known that the tetragonal crystal modification (mod. 2 ) in polybutene-1 transforms into the hexagonal crystal modification (mod. 1) when a fresh sample of this material is allowed to stand after preparation. ${ }^{1}$ Accordingly physical properties related to crystalline structure, such as birefringence, change with time. Yang (Yee) and Stein $^{2,3}$ have investigated the deformation of fresh and aged polybutene-1, and found notable differences between the fresh and the aged samples. The crystalline transformation is also accelerated by stretching. Therefore, the variation of the mechanical properties of this material with time can be fully understood only when this transformation is taken into consideration. However, following the crystal trans-

* Presented at the Seminar on Statistical Mechanics and Spectroscopy of Polymers, Univ. of Mass., Amherst, Mass., U.S.A., August 2-6, 1971.

** To whom all communications should be addressed. formation during stress-strain or stress relaxation tests is usually very difficult, and hence little appreciable research on this line has hitherto been published.

Among the various rheo-optical techniques now available, the simultaneous measurement of infrared dichroism with stress and strain was first developed in our laboratory in $1964^{4}$. Since then it has been applied to many systems, including crystalline polymers, their blends, and block copolymers, demonstrating a broad range of usefulness. This infrared dichroism technique enables us to follow the progress of the crystalline transformation during stress-strain and stress relaxation measurements. The technique also affords information on molecular and crystallite orientation. Thus, the application of this technique to polybutene- 1 may be regarded as one of most powerful means to study the deformation mechanism in this material. The present paper is concerned with the experimental study of the 
deformation process and transformation in polybutene-1.

\section{EXPERIMENTAL}

\section{Material}

Commercial pellets of polybutene-1 placed between two aluminum plates were premelted for $3 \mathrm{~min}$ and then pressed for $5 \mathrm{~min}$ at a pressure of $50 \mathrm{~kg} / \mathrm{cm}^{2}$ in a laboratory press equipped with heating plates. The sample was then removed from the press and quenched by plunging it into an ice water bath. The films thus prepared were designated as fresh samples. The thickness of the films was about $70 \mu$. Fresh samples kept in a desiccator for three weeks or more suffered structural change and were designated as aged samples; their infrared spectra and X-ray diffraction patterns were those characteristic to mod. 1 as reported by Natta, et $a l .^{5,6}$

\section{Measurements}

The crystal transformation from mod. 2 to mod. 1 in polybutene-1 can be detected by several techniques such as X-ray diffraction, ${ }^{1,7}$ infrared absorption, ${ }^{8,9}$ differential thermal analysis, ${ }^{1,10}$ and dilatometry. ${ }^{1,11}$ Among these methods, the infrared absorption method enables us to measure the transformation most quickly.

Natta, et al.,$^{6}$ and Boor, et al.,${ }^{1}$ have already elucidated the infrared spectra corresponding to the two modifications of polybutene-1. From these spectra it can be seen that the bands at $10.81,11.78$, and $12.25 \mu(925,849$, and 816 $\mathrm{cm}^{-1}$ ) are associated with mod. 1 , while another band at $11.03 \mu\left(906 \mathrm{~cm}^{-1}\right)$ is associated with mod. 2 .

In our measurements at no longer than 30 min after film preparation, the fresh samples exhibited the X-ray diffraction intensity curve and infrared spectra for mod. 2. The aged samples, on the other hand, gave those for mod. 1. From our experimental results, the band at $1025 \mathrm{~cm}^{-1}$ was also shown to be associated with mod. 1 .

In order to obtain the relation between the content of mod. 1 crystals and the absorbance $A_{846}$ of the $846 \mathrm{~cm}^{-1}$ band, which is associated with mod. 1, the ratio of $A_{846}$ to the absorbance $A_{1150}$ of the $1150 \mathrm{~cm}^{-1}$ band, used as a measure

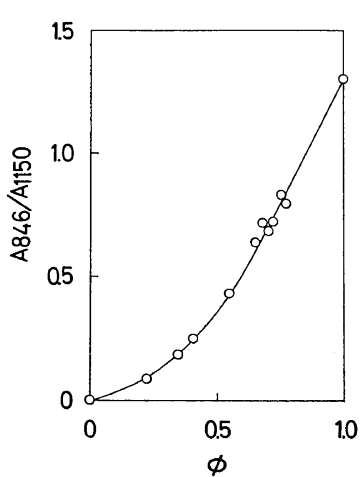

Figure 1. The ratio of the absorbance $A_{846}$ of the $846 \mathrm{~cm}^{-1}$ band to the absorbance $A_{1150}$ of the 1150 $\mathrm{cm}^{-1}$ band against $\Phi$, the degree of crystal transformation.

of thickness, was determined for laminated films of fresh and completely aged samples of different thicknesses. The ratio $A_{846} / A_{1150}$ is plotted against $\Phi$, the degree of crystal transformation, in Figure 1. The degree of crystal transformation $\Phi$ is here defined as the weight fraction of crystals changed from mod. 2 to 1 , and was assumed to be equal to the weight fraction multiplied by the degree of crystallinity of the aged film in the laminates. The curve of Figure 1 provided the standard for infrared measurements of $\Phi$ in the following experiments.

The variation of the infrared dichroic ratio for some key bands characteristic of polybutene1 was measured on the film during stress-strain tests at a constant rate of elongation, 1.64\%/ $\mathrm{min}$, at $20^{\circ} \mathrm{C}$. The apparatus employed was the combination of an infrared spectrometer and a self-recording tensile tester, similar in principle to the Instron Tensile Tester. The details of the apparatus and measuring techniques have been described elsewhere. ${ }^{4}$ The key bands employed were those at 760,905 , and $1025 \mathrm{~cm}^{-1}$.

The refractive index of the sample films was measured by means of an Abbé refractometer at $20^{\circ} \mathrm{C}$, using a small amount of $n$-benzyl alcohol as a contacting liquid.

\section{RESULTS AND DISCUSSION}

\section{Crystal Transformation in Unstrained Samples}

The crystal transformation in a fresh sample at $20^{\circ} \mathrm{C}$ begins at about $30 \mathrm{~min}$ after film pre- 


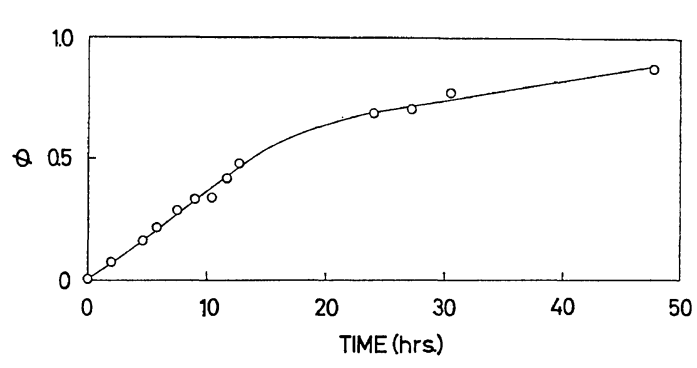

Figure 2. The variation of $\Phi$ with elapsed time, measured from $30 \mathrm{~min}$ after sample preparation.

paration. The variation of $\Phi$ with elapsed time is shown in Figure 2. The elapsed time in this figure is measured from $30 \mathrm{~min}$ after the preparation of the film. $\Phi$ increases with increasing time and reaches about 0.9 at 50 hours and 1.0 at about 3 weeks after sample preparation.

To clarify the time effect upon the crystal transformation, $\log (\log 1 / 1-\Phi)$ is plotted against the logarithm of elapsed time $t$ in Figure 3. The plot gives a straight line having a slope of

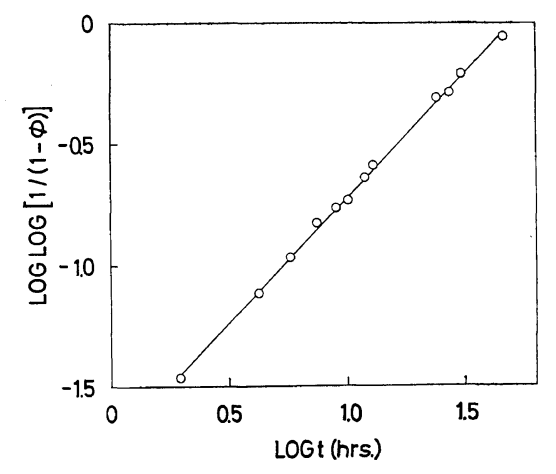

Figure 3. $\log (\log 1 / 1-\Phi) v s . \log$ (elapsed time). unity. We therefore obtain the following relation between $\Phi$ and $t$ :

$$
\log \frac{1}{1-\Phi}=\frac{1}{2.303} k t
$$

It is noteworthy that this equation is quite similar to that for the one-dimensional crystallization rate. ${ }^{12}$ The rate of crystal-crystal transformation is thus described by an equation of the same form as that for crystallization itself. The same relation between $\Phi$ and $t$ is also applicable to the relation between the refractive index and $t$, as shown in the following.

The refractive index is related to the density $\rho$ of the sample by the well-known equation presented by Gladstone and Dale:

$$
\rho=(n-1) M_{\mathrm{u}} / R_{\mathrm{G}}
$$

where $n$ is the refractive index of the sample, $M_{\mathrm{u}}$ the molecular weight of a repeating unit (56.11 for polybutene-1), and $R_{G}$ the so-called Gladstone-Dale molecular refraction, which is calculated to be 31.14 in this case, assuming the additivity of the atomic refraction in the repeating unit. Measurements of the refractive index of polymer films as a function of time have an advantage over the dilatometry and density gradient column methods in providing a rapid trace of the specific volume or the density.

The degree of crystal transformation $\Phi$ was defined above as the weight fraction of crystals transformed from mod. 2 to mod. 1. The amount of mod. 1 crystals formed from the amorphous region must also be taken into account. The two parameters $\Phi_{\mathrm{t}}$ and $\alpha_{\mathrm{t}}$ are thus introduced. $\Phi_{\mathrm{t}}$ is the degree of crystal transformation of the film at

Table I. Several quantities concerned with the crystal transformation

\begin{tabular}{lccc}
\hline & $\begin{array}{c}\text { Fresh } \\
\text { sample }\end{array}$ & $\begin{array}{c}\text { Partially } \\
\text { aged } \\
\text { sample }\end{array}$ & $\begin{array}{c}\text { Aged } \\
\text { sample }\end{array}$ \\
\hline Elapsed time & 0 & $t$ & $\infty$ \\
Degree of crystal transformation & 0 & $\Phi_{t}$ & 1 \\
Weight fraction of mod. 1 transformed from mod. 2 & 0 & $X_{2} \Phi_{t}$ & $X_{2}$ \\
Weight fraction of mod. 2 which remain untransformed & $X_{2}$ & $X_{2}\left(1-\Phi_{t}\right)$ & 0 \\
Weight fraction of mod. 1 formed from the amorphous region & 0 & $\left(X_{1}-X_{2}\right) \alpha_{t}$ & $X_{1}-X_{2}$ \\
Weight fraction of the amorphous phase & $1-X_{2}$ & $1-X_{2}+X_{2} \alpha_{t}-X_{1} \alpha_{t}$ & $1-X_{1}$ \\
\hline
\end{tabular}


the elapsed time $t$, and $\alpha_{\mathrm{t}}\left(0 \leq \alpha_{\mathrm{t}} \leq 1\right)$ is a crystallization parameter. Using these parameters, the weight fractions of mod. 1 crystals transformed from mod. 2, untransformed mod. 2 crystals, and mod. 1 crystals formed from the amorphous region are represented respectively by $X_{2} \Phi_{t}$, $X_{2}\left(1-\Phi_{t}\right)$, and $\left(X_{1}-X_{2}\right) \alpha_{t}$, where $X_{1}$ and $X_{2}$ are respectively the final crystallinity of the aged sample and the initial crystallinity of the fresh sample. These quantities are listed with others in Table I.

$X_{1}$ and $X_{2}$ may be represented as follows:

$$
\begin{aligned}
& X_{1}=\left(V_{\mathrm{A}}-V_{\mathrm{ag}}\right) /\left(V_{\mathrm{A}}-V_{\mathrm{C} 1}\right) \\
& X_{2}=\left(V_{\mathrm{A}}-V_{\mathrm{fr}}\right) /\left(V_{\mathrm{A}}-V_{\mathrm{C} 2}\right)
\end{aligned}
$$

where $V_{\mathrm{A}}, V_{\mathrm{C} 1}$, and $V_{\mathrm{C} 2}$ represent the specific volume of the amorphous region and the mod. 1 and mod. 2 crystal fractions, respectively. $V_{\mathrm{ag}}$ and $V_{\mathrm{fr}}$ represent the specific volumes of the aged and fresh samples respectively.

The specific volumes of the sample film at the elapsed times $t=0, t=t$, and $t=\infty, v_{0}, v_{\mathrm{t}}$, and $v_{\infty}$, are given by

$$
\begin{gathered}
v_{0}=V_{\mathrm{A}}\left(1-X_{2}\right)+V_{\mathrm{C} 2} X_{2} \\
v_{\mathrm{t}}=V_{\mathrm{A}}\left(1-X_{2}-X_{1} \alpha_{\mathrm{t}}+X_{2} \alpha_{\mathrm{t}}\right) \\
+V_{\mathrm{C} 1}\left(X_{2} \Phi_{\mathrm{t}}+X_{1} \alpha_{\mathrm{t}}-X_{2} \alpha_{\mathrm{t}}\right)+V_{\mathrm{C} 2}\left(X_{2}-X_{2} \Phi_{\mathrm{t}}\right) \\
v_{\infty}=V_{\mathrm{A}}\left(1-X_{1}\right)+V_{\mathrm{C} 1} X_{1}
\end{gathered}
$$

Consequently, we obtain

$$
\begin{aligned}
& \frac{v_{0}-v_{\mathrm{t}}}{v_{0}-v_{\infty}} \\
= & \frac{V_{\mathrm{A}}\left(X_{1}-X_{2}\right) \alpha_{\mathrm{t}}-V_{\mathrm{C} 1}\left(X_{2} \Phi_{\mathrm{t}}+X_{1} \alpha_{\mathrm{t}}-X_{2} \alpha_{\mathrm{t}}\right)+V_{\mathrm{C} 2} X_{2} \Phi_{\mathrm{t}}}{V_{\mathrm{A}}\left(X_{1}-X_{2}\right)+V_{\mathrm{C} 2} X_{2}-V_{\mathrm{C} 1} X_{1}}
\end{aligned}
$$

If we assume a simple case where $X_{1}=X_{2}$ and thus $\alpha_{\mathrm{t}}$ may be neglected, eq 8 reduces to

$$
\left(v_{0}-v_{\mathrm{t}}\right) /\left(v_{0}-v_{\infty}\right)=\Phi_{\mathrm{t}}
$$

Replacing $\Phi$ in eq 1 with $\Phi_{t}$ of eq 9 , we obtain

$$
\log \left(\log \frac{v_{0}-v_{\infty}}{v_{t}-v_{\infty}}\right)=\log (k / 2.303)+\log t
$$

This equation indicates that a plot of the lefthand side against $\log t$ should give a straight line, whose slope is unity. In Figure 4, such a plot is made for the refractive index data.

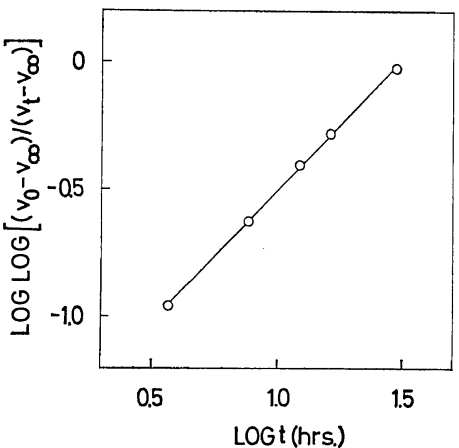

Figure 4. $\log \left\{\log \left(v_{0}-v_{\infty}\right) /\left(v_{t}-v_{\infty}\right)\right\} v s . \log$ (elapsed time).

The plot shows that the experimental results satisfy eq 10 well, and that the relationship of eq 1 is thus applicable also to data derived from measurements of the refractive index as a function of elapsed time.

The values of $k$ can be evaluated from the intercepts of the straight line in Figure 3 and Figure 4. The $k$ value obtained from the infrared data (Figure 3) is somewhat larger than that obtained from the refractive index data (Figure 4). One of the reasons for this difference is probably that the refractive index is determined mainly by the crystal transformation at or near the surface of the sample film. This is supported by the observation of Luongo, et al. ${ }^{8}$, who found that the crystal transformation at or near the surface of polybutene-1 film is always greater than that in the inner part.

Stress-Strain Behavior and Crystal Transformation

As demonstrated above, a fresh sample of polybutene-1 suffers crystal transformation even when it is not stretched. It has also been known that the crystal transformation is accelerated by stretching the sample. However, the relation between the crystal transformation and the mechanical behavior has not been fully investigated hitherto. We therefore measured the mechanical and orientation behavior at the same time by the use of rheo-optical techniques.

Stress-strain curves for film specimens having different initial degrees of crystal transformation $\Phi_{0}$ were obtained under a constant rate of elongation $(1.64 \% / \mathrm{min})$, and are shown in Figure 5. Except for the case of $\Phi_{0}=1$, no macroscopic necking was observed during the tests. For the 


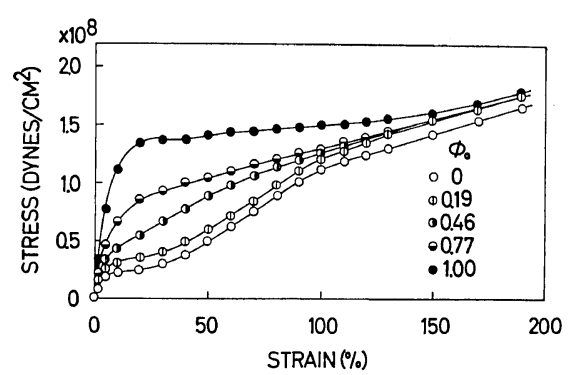

Figure 5. Stress-strain curves for film specimens having different initial degrees of crystal transformation.

sample with $\Phi_{0}=1.0$, necking was observed at the strain of $30 \%$. The curves in this figure differ very much at low strains, but they draw closer at higher strains. The lower $\Phi_{0}$ is, the lower is the yield stress. In other words, aged samples need more energy to deform up to the yield point than do fresh ones. This difference below the yield point is due to the difference between fresh and aged samples in resistance to the disintegration of spherulites. On the other hand, the rate of increase in the stress beyond the yield point is lower for the aged samples than for the fresh ones.

It was surmised that the great differences in stress and rate of increase in stress beyond the yield point were associated with the crystal transformation. Therefore the variation of the degree of crystal transformation with strain was measured simultaneously with the stress-strain behavior. The results are shown in Figure 6. As is evident in this figure, $\Phi$ for the fresh and incompletely aged samples increases with increasing strain. The lower the initial degree of transformation $\Phi_{0}$, the greater the variation of

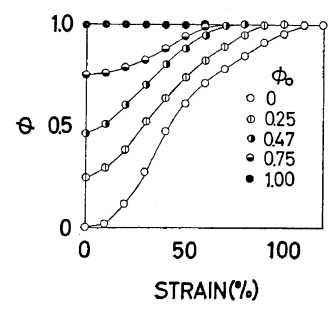

Figure 6. The variation of the degree of crystal transformation with strain during stress-strain measurements.
$\Phi$ with strain. It follows from these results that one must pay attention to the crystal transformation phenomenon when interpreting the stressstain curves of polybuten-1. $\Phi$ for the fresh sample $\left(\Phi_{0}=0\right)$ increases slowly up to about $10-\%$ elongation, but then very rapidly up to about 50-\% elongation, and reaches its ceiling value, i.e., 1 , at about $105-\%$ elongation. Similar behavior is observed for the other samples, excepting the fully aged one. The higher the $\Phi_{0}$ value, the smaller the strain at which $\Phi$ reaches the ceiling value.

Next, the dichroic ratio $D_{760}(|| / \perp)$ of the 760 $\mathrm{cm}^{-1}$ band was determined during stree-strain measurements; it is plotted against strain in Figure 7. Since the band at $760 \mathrm{~cm}^{-1}$ is associated with the crystallite orientation of both modifications 1 and 2, $D_{760}$ can serve as a measure of

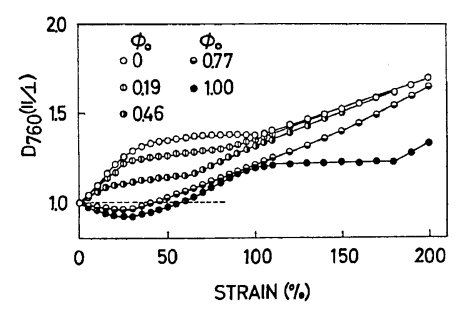

Figure 7. The variation of the dichroic ratio $D_{760}$ $\left(11 / \perp\right.$ ) of the $760 \mathrm{~cm}^{-1}$ band with strain, determined during stress-strain measurements.

total crystallite orientation. As is seen from the figure, $D_{760}$ for the fresh sample $(\Phi=0)$ increases with increasing strain up to about 40-\% strain, remains almost constant from $40-\%$ to $105-\%$ strain, and then increases rapidly again at larger strains. It is noteworthy that the strain of $105-\%$ coincides with the strain at which $\Phi$ reaches its ceiling value ( $c f$., Figure 6). For the sample with $\Phi_{0}=0.46, D_{760}$ increases with increasing strain up to about $20-\%$ and exhibits a plateau extending to about $60-\%$ strain. The height of this plateau is lower than that for the fresh sample with $\Phi_{0}=0$.

On the other hand, for the sample having $\Phi_{0}=1.0$ and $0.77, D_{760}$ decreases at first and then increases after passing through a minimum at about $30-\%$ strain. The plateau observed for the aged sample between $110-\%$ and $180-\%$ strain 


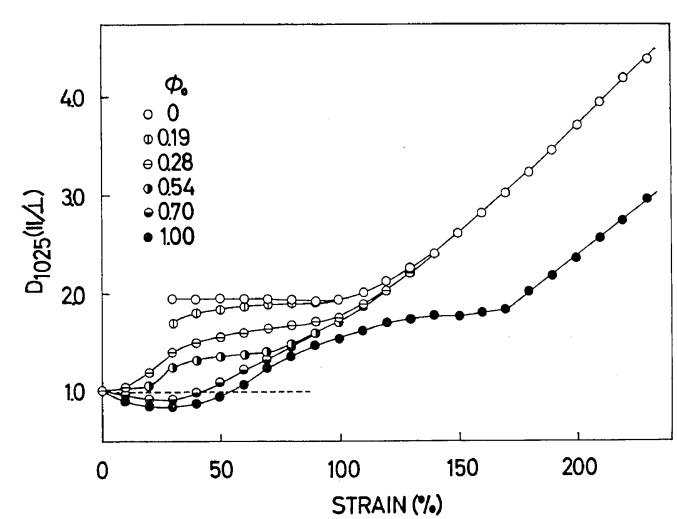

Figure 8. The variation of the dichroic ratio $D_{1025}$ $(I / / \perp)$ for the $1025 \mathrm{~cm}^{-1}$ band with strain during stress-strain measurements.

is only an apparent plateau, due to partial necking. It is noteworthy that the strain at which $D_{760}$ takes its minimum coincides with the strain at which macroscopic necking starts (compare Figure 5 with Figure 7).

The following two propositions may be considered as reasons why a plateau is observed in the $D_{760}$ vs. strain curves for partially aged samples: (i) the orientation of crystallites, both in mod. 1 and mod. 2, does not change during deformation in this region; or (ii) the orientation of mod. 1 crystals increases while that of mod. 2 crystals decreases, or vice versa, so that the total crystallite orientation does not change. In order to determine which is the true explanation, the dichroic ratio $D_{1025}(|| / \perp)$ for the $1025 \mathrm{~cm}^{-1}$ band was measured as a function of strain and is shown in Figure 8. Since the band at 1025 $\mathrm{cm}^{-1}$ is a parallel band and is associated only with mod. 1 crystals, the dichroic ratio $D_{1025}$ $(\mid / / \perp)$ is directly related to the degree of orientation of mod. 1 crystals. The variation of $D_{1025}$ with strain for the samples having $\Phi_{0}=1.0$ and 0.7 are similar to those of $D_{760}$, with $D_{1025}$ taking a minimum at about $30-\%$ strain. Similarly, the variation of $D_{1025}$ with strain for the fresh and partially aged samples is very similar to that of $D_{760}$, though $D_{1025}$ could not be measured accurately at the smaller strains. The conclusion is that the plateau observed in the $D v s$. strain curves for the fresh and other samples is due to the fact that the orientation of crystals in both mod. 1 and mod. 2 does not change with strain in the intermediate region.

These results suggest that the orientation of mod. 2 crystals predominates at small strains, and that the oriented crystals show a higher degree of orientation or higher $D_{1025}$ even after they have changed into mod. 1.

For comparison, the variation of the dichroic ratio $D_{905}(\perp / \|)$ for the $905 \mathrm{~cm}^{-1}$ band with strain was also measured. This band is a perpendicular band associated only with the orientation of mod. 2 crystals, and therefore serves as a measure of the orientation of these crystals. The results are shown in Figure 9. It is evident

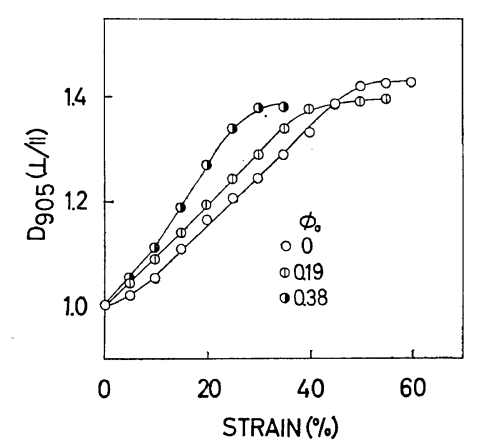

Figure 9. The variation of the dichroic ratio $D_{905}$ $(\perp / 11)$ for the $905 \mathrm{~cm}^{-1}$ band with strain during stress-strain measurements.

from this figure that $D_{905}$ increases almost linearly with increasing strain and levels off at larger strains. The differences between samples are rather small.

To summarize the above discussion, the stressstrain behavior of the fresh sample, for example, can be divided into the following three processes:

(i) $0 \leq$ strain $\gamma \leq 30-\%$ : Both orientation and transformation of crystals take place. The orientation of mod. 2 crystals proceeds, and crystals changes into mod. 1 .

(ii) $30 \leqslant \gamma \leqslant 105-\%$ : Crystal transformation occurs predominantly, and the orientation remains almost constant. The crystal transformation results in more extended chain conformation, since the molecular chains are more extended in mod. 1 than in mod. 2 crystals. Therefore, the orientation due to external straining compensates for the disorientation due to self-induced extension during the crystal transformation from 
mod. 2 to mod. 1 .

(iii) $r \geq 105-\%$ : Crystal transformation is complete, and orientation increases again.

On the other hand, the deformation process in the aged sample is very different from that in the fresh sample. That is to say, the crystallite orientation initially decreases with strain. The strain at which the dichroic ratio takes its minimum coincides with the strain at the yield point. Such behavior of aged polybutene-1 is very similar to that of polypropylene described elsewhere. ${ }^{13}$ Below the yield point, spherulites or superstructure consisting of lamellae show elastic deformation, and the lamella axes orient in the direction of elongation. At the yield point, the spherulites disintegrate but show great resistance, or a high yield value. Beyond the yield point, the disintegrated spherulites or superstructure show irrecoverable deformation due to the unfolding of folded chains, which orient in the direction of stretching as the strain increases.

On the other hand, the spherulites or superstructures of the fresh sample consist of crystallites in mod. 2 and are easily disintegrated; the yield value is very low. Moreover, unfolding of folded chains also occurs very easily.

The deformation process in samples having $\Phi_{0}$ between 1 and 0 is intermediate between these two extreme cases.

\section{REFERENCES}

1. J. Boor, Jr. and J. C. Mitchell, J. Polym. Sci., Part A, 1, 59 (1963).

2. R. Yang and R. S. Stein, J. Polym. Sci., Part A-2, 5, 939 (1967).

3. R. Y. Yee and R. S. Stein, J. Polym. Sci., Part $A-2,8,1661$ (1970).

4. S. Onogi, H. Kawai and T. Asada, Kobunshi Kagaku (Chem. High Polymers), 21, 746 (1964).

5. G. Natta, P. Corradini and I. W. Bassi, Makromol. Chem., 21, 240 (1956).

6. G. Natta, P. Corradini and I. W. Bassi, Nuovo Cimento Suppl., 15, 52 (1960).

7. F. Sakaguchi, Sen-i to Kogyo (Fiber Science and Industry, Japan), 1, 402 (1968).

8. J. P. Luongo and R. Salovey, J. Polym. Sci., Part A-2, 4, 997 (1966).

9. G. Goldback and G. Peitsher, J. Polym. Sci., Part B, 6, 783 (1968).

10. I. D. Rubin, J. Polym. Sci., Part A, 3, 3803 (1965).

11. J. Powers, J. D. Hoffmann, J. J. Weeks and F. A. Quinn, Jr., J. Res. NBS, 69A, 335 (1965).

12. I. D. Lauritzen and J. D. Hoffmann, J. Res. NBS, 64A, 73 (1960).

13. S. Onogi, T. Asada, and T. Takaki, Zairyo, 16. 746 (1967). 